Schmerz $2023 \cdot 37: 38-46$

https://doi.org/10.1007/s00482-021-00619-5

Angenommen: 15. Dezember 2021

Online publiziert: 17. Januar 2022

○ Der/die Autor(en) 2022

\section{Postoperatives Schmerzerleben nach proximaler Femurfraktur bei Demenz}

\author{
Jens Felix Wagner' $\cdot$ Henning Cuhls ${ }^{2} \cdot$ Martin Mücke $^{3,4} \cdot$ Rupert Conrad ${ }^{5}$. \\ Lukas Radbruch $^{2,6} \cdot$ Roman Rolke $^{7}$ \\ ${ }^{1}$ Akutgeriatrie und Tagesklinik, Helios Klinikum Bonn/Rhein-Sieg, Bonn/Rhein-Sieg, Deutschland; ${ }^{2}$ Klinik \\ und Poliklinik für Palliativmedizin, Universitätsklinikum Bonn, Bonn, Deutschland; ${ }^{3}$ Institut für Digitale \\ Allgemeinmedizin, Medizinische Fakultät, RWTH Aachen University, Aachen, Deutschland; ${ }^{4}$ Zentrum für \\ Seltene Erkrankungen Aachen (ZSEA), Medizinische Fakultät, RWTH Aachen University, Aachen, \\ Deutschland; ${ }^{5}$ Klinik und Poliklinik für Psychosomatische Medizin und Psychotherapie, \\ Universitätsklinikum Bonn, Bonn, Deutschland; ${ }^{6}$ Zentrum für Palliativmedizin, Helios Klinikum \\ Bonn/Rhein-Sieg, Bonn/Rhein-Sieg, Deutschland; ' ${ }^{7}$ Klinik für Palliativmedizin, Medizinische Fakultät, \\ RWTH Aachen University, Aachen, Deutschland
}

Hintergrund: Die vorliegende Studie verfolgte das Ziel, das postoperative Schmerzerleben bei kognitivem Defizit unter besonderer Berücksichtigung der sensorischen oder affektiven Schmerzqualität zu erfassen.

Methodik: 19 Patienten mit normaler Kognition bis hin zu kognitiven Auffälligkeiten im Screening-Fragebogen DemTect wurden bezüglich ihres postoperativen Schmerzerlebens nach proximaler Femurfraktur untersucht. Als Untersuchungsinstrumente kamen die numerische Ratingskala (NRS), der Kognitionsfragebogen DemTect, der Schmerzempfindungsfragebogen (SES) sowie eine quantitative sensorische Testung (QST) zum Einsatz.

Ergebnisse: Das Alter der Patienten lag im Mittel \pm SD bei $83,8 \pm 10,0$ Jahren. Von 19 Patienten lagen bei $6(31,6 \%)$ normale kognitive Fähigkeiten vor, bei 4 Patienten $(21,1 \%)$ ergaben sich Hinweise auf leichte kognitive Einschränkungen, bei 9 Patienten (47,4\%) ergab sich der Verdacht auf Vorliegen einer Demenz. Die mittlere postoperative Schmerzintensität (NRS) betrug 4,0 $(1,6)$. Die berichteten Schmerzintensitäten unterschieden sich bei vergleichbarer analgetischer Therapie nicht zwischen den drei Patientengruppen mit unterschiedlicher kognitiver Beeinträchtigung und den ersten drei postoperativen Behandlungstagen. Es zeigten sich zwischen den Gruppen keine statistisch signifikanten Unterschiede für die sensorischen oder affektiven Summenscores der Schmerzempfindungsskala.

Die QST-Parameter Tiefenschmerz (PPT), oberflächlicher mechanischer Schmerz nach Nadelreiz (MPT) sowie die oberflächliche Sensibilität für leichte Berührungsreize (MDT) zeigten eine signifikant gesteigerte Empfindlichkeit der operierten Seite. Für das Vibrationsempfinden (VDT) konnten keine Unterschiede zwischen operierter und gesunder Extremität nachgewiesen werden.

Diskussion: Das postoperative Schmerzerleben unterscheidet sich nicht zwischen Patienten mit normaler und deutlich eingeschränkter Kognition. Die quantitative sensorische Testung zeigte mechanische Hyperalgesien im operierten Areal. Die Studie weist auf die Wichtigkeit einer adäquaten postoperativen Schmerzversorgung auch bei Betroffenen mit Demenz hin.

\section{Schlüsselwörter}

Schmerzempfindungsskala · Quantitative sensorische Testung · Somatosensorisches Nervensystem · Sensibilitätsprüfung · Geriatrie 


\section{Hintergrund und Fragestellung}

„Altern", "Demenz" und "Schmerz" sind Begriffe, die in unserer Gesellschaft zunehmend an Bedeutung gewinnen. Bei einer prognostizierten Abnahme der Gesamtbevölkerung von 82 Mio. in 2008 auf 73,8 Mio. in 2040 steigt der Anteil älterer Menschen über 65 von 20,4 Mio. auf 32,1 Mio. an (Statistisches Bundesamt, 2009). Mit der höheren Lebenserwartung nimmt die Prävalenz von Krankheiten und funktionellen Einschränkungen zu. So führen beispielsweise Osteoporose und vermehrte Sturzneigung bedingt durch funktionellen Abbau, Sehschwäche, Muskelschwäche, Gang- und Gleichgewichtsstörungen sowie zentral wirksame Medikamente zu erhöhter Frakturneigung. Das Risiko, eine Femurfraktur zu erleiden, ist bei dementen Patienten bis zu dreimal höher als bei älteren Patienten mit normalem kognitivem Status [11, 41].

Die angemessene Beurteilung von Schmerzen bei Menschen mit mittelgradiger Demenz stellt für die betreuenden Pflegefachkräfte und Ärzte eine Herausforderung dar [12, 22]. So werden stärkere Schmerzen eher unterschätzt, leichtere Schmerzen eher überschätzt $[8,40]$. Patienten mit Alzheimer-Demenz erhielten in Pflegeeinrichtungen nur ein Drittel der Morphindosis im Vergleich mit Patienten, die einen normalen kognitiven Status aufwiesen [7, 10]. Nach operativer Versorgung einer Femurfraktur zeigten demente Patienten mehr Schmerzen als Vergleichspersonen [30]. Als Folge eines unzureichenden Schmerzmanagements bei Demenz können sich Schlafstörungen, Depressionen und Ängste entwickeln [25]. Auch die Entwicklung eines Delirs kann durch einen intensiven postoperativen Schmerzzustand begünstigt werden [27]. Eine adäquate Schmerztherapie kann zu weniger postoperativen Komplikationen, einer kürzeren Verweildauer im Krankenhaus, einer besseren Lebensqualität sowie reduzierter Morbidität und Mortalität führen $[29,36]$.

Bislang fehlen Untersuchungen zum postoperativen Schmerzerleben von dementen im Vergleich mit kognitiv unbeeinträchtigten Patienten.

Hieraus leiten sich die konkreten Fragestellungen der vorliegenden Studie ab:
1. Zeigen Betroffene mit Demenz im Vergleich mit kognitiv Unbeeinträchtigten eine veränderte postoperative Stärke der erlebten Ruheschmerzen?

2. Haben Menschen mit Demenz ein verändertes sensorisches oder affektives Schmerzerleben?

3. Wie unterscheidet sich das sensorische Profil (hier erhoben mit mechanischen Testreizen) bei Patienten ohne und mit kognitivem Defizit im Vergleich der operierten und kontralateralen Seite nach Hüftendoprothese?

\section{Methodik}

In der vorliegenden Studie wurde das postoperative Schmerzerleben nach Femurfraktur untersucht. Anhand des Demenz-Screening-Fragebogens "DemTect" [20] wurden die Patienten in drei Kognitionsgruppen kategorisiert: normale Kognition, leichtes kognitives Defizit und Verdacht auf Demenz. Mittels quantitativer sensorischer Testung (QST) wurde die Schmerzempfindlichkeit der operierten Hüfte mit dem nicht operierten, kontralateralen Bereich verglichen. Ein modifizierter SES-Schmerzfragebogen (SES = Schmerzempfindungsskala) [15] wurde verwendet, um die Patienten zum aktuellen postoperativen Schmerzerleben mithilfe sensorischer und affektiver Deskriptoren zu befragen.

\section{Patientenkollektiv}

In die Studie wurden 19 Patienten mit einer proximalen Femurfraktur nach ausführlicher schriftlicher und mündlicher Aufklärung eingeschlossen, die eine operative Versorgung in Form eines endoprothetischen Ersatzverfahrens oder eines Osteosyntheseverfahrens erhielten. Die Studie mit der Ethik-Antragsnummer 261/12 an der Medizinischen Fakultät der Universität Bonn erfolgte nach den Grundsätzen guter klinischer Praxis (GCP) unter Einhaltung der Deklaration von Helsinki [2].

Einschlusskriterien waren ein Alter über 60 Jahre, eine frisch aufgetretene proximale Femurfraktur, Ruheschmerzen $\geq 2$ von 10 auf der numerischen Ratingskala (NRS) sowie die schriftliche Einwilligung zur Teilnahme an der Studie durch den Patienten oder im Fall reduzierter Kognition durch einen Bevollmächtigten. Ausschlusskriterien waren Migräne oder Rückenschmerzen in den letzten acht Wochen oder Erkrankungen, die zu einer Veränderung der Sensibilität gegenüber den Testreizen führen, sowie Hautveränderungen, die eine Anwendung der QST-Geräte einschränken könnten. Außerdem wurden Patienten mit einem postoperativen Delir von der Studienteilnahme ausgeschlossen.

\section{Studienablauf}

Durchgeführt wurde die Untersuchung in den Abteilungen für Chirurgie und Geriatrie des Helios Klinikums (vormals Malteser Krankenhaus) Bonn/Rhein-Sieg vom 11.03.2013 bis zum 03.06.2013. Die Untersuchungen erfolgten im Rahmen einer klinischen monozentrischen Beobachtungsstudie. Alle Patienten erhielten nach dem operativen Eingriff eine auf die individuelle Schmerzangabe abgestimmte und nicht standardisierte analgetische Therapie. Keiner der Patienten erhielt ein Regionalanästhesieverfahren. Alle Testungen (NRS, DemTect, SES, QST) erfolgten durch denselben Untersucher (JFW) innerhalb der ersten drei postoperativen Tage zwischen 10:00 und 16:00 Uhr.

Anamnese, klinische Untersuchung und Erfassung der aktuellen

Schmerzstärke

Nach Anamneseerhebung fanden eine symptombezogene klinische Untersuchung (Wundinspektion) sowie die Beurteilung des klinischen Gesamtzustands statt. Im Anschluss erfolgte die Abfrage der zum Untersuchungszeitpunkt empfundenen Ruheschmerzen mithilfe einer numerischen Ratingskala (NRS

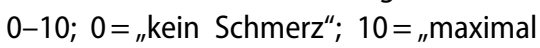
vorstellbarer Schmerz").

\section{Erfassung des kognitiven Status mittels DemTect-Fragebogen}

Der DemTect-Fragebogen erfasst verschiedene kognitive Dimensionen: verbales Gedächtnis, Wortflüssigkeit, intellektuelle Flexibilität und Aufmerksamkeit [20]. Die Rohwerte des Tests wurden in altersbezogene Testwerte umcodiert. Dabei dient der DemTect als Screening und nicht als dia- 
gnostisches Instrument mit dem Ergebnis eines Summenscores: $0-8=$ Verdacht auf Demenz; 9-12= leichte kognitive Beeinträchtigung; $13-18=$ normale Kognition.

\section{Schmerzempfindungsskala (SES)}

Der hier verwendete modifizierte SESFragebogen erfasst die Ausprägung von 24 Schmerzdeskriptoren zur Charakterisierung des aktuellen Schmerzerlebens [15]. Der Patient bewertet jeden Deskriptor im affektiven und sensorischen Teil der Schmerzempfindungsskala auf einer 4-Punkte-Skala. Als Anpassung zum Originalfragebogen wurde in dieser Untersuchung das Fehlen eines Schmerzmerkmals mit dem Wert "0" (,trifft nicht $\mathrm{zu}^{\prime \prime}$ ) bewertet. Eine maximale Ausprägung wurde mit der Zahl "3“ (,trifft genau zu“) bewertet. Die Werte für die Globaldimension SES-affektiv und SES-sensorisch wurden anschließend durch Summation der Testwerte der einzelnen Deskriptoren ermittelt.

\section{Quantitative sensorische Testung (QST)}

Die quantitative sensorische Testung ist eine formalisierte und standardisierte klinische Sensibilitätsprüfung mit kalibrierten Testreizen [31]. Die Untersuchung wurde anhand des DFNS-Protokolls (Deutscher Forschungsverbund Neuropathischer Schmerz) durchgeführt [38, 39]. Da den für die Operation anstehenden Patienten für die QST-Untersuchung kein Transport in ein Untersuchungslabor zumutbar war, wurden nur mechanische Tests im Patientenzimmer durchgeführt und auf eine schwer transportable PCgestützte Thermotestung verzichtet. Die Testung wurde am ventralen Oberschenkel am Übergang vom proximalen zum mittleren Drittel der Strecke zwischen der Spina iliaca anterior superior und der Patella durchgeführt. Die Testung erfolgte in einem ausreichenden Abstand von ca. $5-8 \mathrm{~cm}$ zur Operationswunde. Zunächst erfolgte als Referenz die QSTUntersuchung der gesunden Seite (Kontrollareal), anschließend der operierten Seite (Testareal). Dabei kamen vier Verfahren mit mechanischen Testreizen zum Einsatz: die mechanische Detektions- schwelle (MDT = mechanical detection threshold) wurde mittels eines standardisierten Sets modifizierter von „Von Frey“Filamente bestimmt (Optihair ${ }_{2}$-Set, Marstock, Deutschland; 0,25 bis $512 \mathrm{mN}$ ) [13]. Die mechanische Detektionsschwelle wurde als geometrischer Mittelwert aus fünf gerade überschwelligen und fünf gerade unterschwelligen Messwerten bestimmt.

Die Untersuchung der mechanischen Schmerzschwelle (MPT = mechanical pain threshold) erfolgte mit Nadelreizstimulatoren (Pinpricks) [4]. Ziel war die Erfassung des ersten schmerzhaften Nadelreizes, der als "spitz" oder "pieksend“ empfunden wurde. Dabei wurden Nadelreizstimulatoren mit flacher Spitze $(0,25 \mathrm{~mm}$ Durchmesser; 8 bis $512 \mathrm{mN}$ ) verwendet. Als Schmerzschwelle wurde der geometrische Mittelwert von jeweils fünf gerade überund unterschwelligen Reizstärken berechnet.

Die Vibrationsdetektionsschwelle (VDT = vibration detection threshold) wurde mittels einer standardisierten $64-\mathrm{Hz}-$ Stimmgabel $[5,17]$ untersucht, die ebenfalls im Abstand von $5-8 \mathrm{~cm}$ zur Op.Wunde mit sehr leichtem Druck auf die Haut aufgebracht wurde. Im Unterschied zum DFNS-QST-Protokoll wurde VDT entsprechend nicht über einer knöchernen Prominenz untersucht. VDT wurde als gerade nicht mehr wahrgenommene Vibrationsempfindung in $\mathrm{x} / 8$ als arithmetischer Mittelwert von drei Serien absteigender Stimulusintensitäten angegeben.

Die Druckschmerzschwelle (PPT = pressure pain threshold) wurde mittels eines Druckalgometers (Algometer, Somedic, Schweden) bestimmt. Mit einer gummierten Kontaktfläche von $1 \mathrm{~cm}^{2}$ wurden drei Serien zunehmender Reizintensitäten $\left(0,5 \mathrm{~kg} / \mathrm{cm}^{2 *} \mathrm{~s}\right.$, entspricht ca. $\left.50 \mathrm{kPa} / \mathrm{s}\right)$ bis maximal $20 \mathrm{~kg} / \mathrm{cm}^{2}$ (ca. maximal $2000 \mathrm{kPa}$ ) über der zu untersuchenden Körperregion aufgebracht. Als Druckschmerzschwelle wurde die aus drei Messungen gemittelte Reizintensität der jeweils ersten schmerzhaft drückenden Wahrnehmung bestimmt $[23,37]$.

\section{Statistische Auswertungen}

Die Auswertung der Schmerzschätzungen sowie der QST-Befunde erfolgte mithilfe des Programms STATISTICA 7.1
(Statsoft Europe, Hamburg, Deutschland). Aufgrund der nicht-parametrischen Verteilung einzelner QST-Parameter wurde nach sekundärer Normalisierung der Daten durch eine Log-Transformation eine Varianzanalyse (ANOVA) für multivariate Analysen durchgeführt. Anschließend folgten Post-Hoc-Tests (LSD-Post-HocTest = least significant difference), um einzelne Kontraste darzustellen. Die QSTDaten wurden grafisch als Rohdaten-Mittelwerte (SEM) dargestellt. Neben dem Vergleich der operierten und Kontrollseite wurde hier die postoperative Schmerzempfindlichkeit auch im Vergleich der Kognitionsgruppen analysiert. Die Zuteilung der Patienten zu den drei Kognitionsgruppen wurde nach Anzahl der erreichten Punkte im DemTect getroffen. Die Daten des SES-Fragebogens wurden deskriptiv und zusätzlich mittels getrennter Faktorenanalysen für die affektiven und sensorischen Schmerzdeskriptoren untersucht. Die Datenextraktion erfolgte mit der Varimax-Methode (einfache Datenrotation). Berücksichtigt wurden nur Faktoren mit einem Eigenwert $>1$.

\section{Ergebnisse}

\section{Patienten}

Das Alter der Patienten lag im Mittel \pm SD bei $83,8 \pm 10,0$ Jahren. $84 \%$ der Patienten waren Frauen, $16 \%$ waren Männer. Von den 19 Studienpatienten wurden elf $(57,9 \%)$ am ersten, zwei $(10,5 \%)$ am zweiten und sechs $(31,6 \%)$ am dritten postoperativen Tag untersucht. Bei 16 Patienten lag eine mediale Schenkelhalsfraktur vor $(84,2 \%)$, bei zwei Patienten eine subtrochantäre Femurfraktur (10,5\%), bei einem Patienten handelte es sich um eine pertrochantäre Femurfraktur (5,3\%). In zehn Fällen $(52,6 \%)$ war die Fraktur auf der rechten Seite lokalisiert, in neun Fällen (47,4\%) auf der linken. In der $\bullet$ Tab. 1 sind die Patientendaten nach Kognitionsgruppen mit Alter, Geschlecht, aktuellem NRS und Schmerzmedikation zusammengefasst.

\section{Postoperatives medikamentöses Schmerzmanagement}

Alle Patienten erhielten Nichtopioid-Analgetika. Es fanden sich dabei keine 
Tab. 1 Patientenübersicht nach Kognitionsgruppen und eingesetzten Schmerzmitteln

\begin{tabular}{|c|c|c|c|c|c|c|}
\hline $\begin{array}{l}\text { Kognition } \\
\text { Gruppe }\end{array}$ & $\begin{array}{l}\text { Kognition Punkte- } \\
\text { zahl DemTect }\end{array}$ & Alter & Geschlecht & NRS & Opioide (Tagesdosis) & Nichtopioid-Analgetika (Tagesdosis) \\
\hline 1 & 14 & 61 & $\mathrm{~m}$ & 7 & - & Ibuprofen $1800 \mathrm{mg}$ \\
\hline 1 & 15 & 62 & w & 5 & - & Ibuprofen $1800 \mathrm{mg}$ \\
\hline 1 & 18 & 70 & w & 3 & Tilidin $200 \mathrm{mg}$ & Ibuprofen $1800 \mathrm{mg}$ \\
\hline 1 & 18 & 75 & w & 4 & - & Metamizol $2 \mathrm{~g}$ \\
\hline 1 & 14 & 84 & $\mathrm{~m}$ & 3 & - & Metamizol 1,5g \\
\hline 1 & 16 & 88 & w & 7 & - & Ibuprofen $1800 \mathrm{mg}$, Metamizol $2 \mathrm{~g}$ \\
\hline 2 & 10 & 83 & w & 2 & - & Ibuprofen $1800 \mathrm{mg}$, Metamizol 4g \\
\hline 2 & 9 & 86 & w & 2 & Tilidin $150 \mathrm{mg}$ & Metamizol 1,5g \\
\hline 2 & 10 & 91 & $\mathrm{~m}$ & 4 & Tilidin $100 \mathrm{mg}$ & Metamizol 4g \\
\hline 2 & 10 & 92 & w & 5 & Tilidin $100 \mathrm{mg}$ & Metamizol $2 \mathrm{~g}$ \\
\hline 3 & 8 & 83 & w & 4 & Tilidin $100 \mathrm{mg}$ & Metamizol 2,5g \\
\hline 3 & 7 & 83 & w & 6 & - & Ibuprofen $1200 \mathrm{mg}$, Metamizol $2 \mathrm{~g}$, Diclofenac $150 \mathrm{mg}$ \\
\hline 3 & 4 & 85 & w & 2 & Tilidin $100 \mathrm{mg}$ & Ibuprofen $1800 \mathrm{mg}$ \\
\hline 3 & 6 & 89 & w & 4 & - & Metamizol $2 \mathrm{~g}$ \\
\hline 3 & 5 & 89 & w & 3 & Tilidin $200 \mathrm{mg}$ & Metamizol 4g \\
\hline 3 & 8 & 92 & w & 2 & - & Ibuprofen 1200 mg, Diclofenac 75 mg \\
\hline 3 & 8 & 93 & w & 4 & Hydromorphon 24 mg & Flupirtin, $200 \mathrm{mg}$, Metamizol 2,5g \\
\hline 3 & 8 & 93 & w & 5 & Tilidin $300 \mathrm{mg}$ & Ibuprofen 1800 mg, Metamizol $2 \mathrm{~g}$ \\
\hline 3 & 4 & 93 & w & 4 & - & Metamizol $2 \mathrm{~g}$ \\
\hline
\end{tabular}

Tab. 2 ANOVA QST-Parameter

\begin{tabular}{|c|c|c|c|c|c|c|}
\hline \multirow[b]{2}{*}{ QST-Parameter } & \multicolumn{2}{|c|}{$\begin{array}{l}\text { Faktor 1: Vergleich ope- } \\
\text { rierte vs. Kontrollseite }\end{array}$} & \multicolumn{2}{|c|}{$\begin{array}{l}\text { Faktor 2: Kognitionsgrup- } \\
\text { pe (normal, MCI, Demenz) }\end{array}$} & \multicolumn{2}{|c|}{$\begin{array}{l}\text { Interaktion Fak- } \\
\text { toren } 1 * 2\end{array}$} \\
\hline & F-Wert & $p$-Wert & F-Wert & $p$-Wert & F-Wert & $p$-Wert \\
\hline PPT & 47,9 & $<0,001$ & 0,23 & n.s. & 0,59 & n.s. \\
\hline MDT & 49,6 & $<0,001$ & 1,08 & n.s. & 0,23 & n.s. \\
\hline MPT & 20,5 & $<0,001$ & 2,41 & n.s. & 0,46 & n.s. \\
\hline VDT & 5,0 & $<0,05$ & 0,44 & n.s. & 0,06 & n.s. \\
\hline \multicolumn{7}{|c|}{$\begin{array}{l}\text { Legende: PPT pressure pain threshold (Druckschmerzschwelle), } M D T \text { mechanical pain detection } \\
\text { threshold (taktile Detektionsschwelle), } M P T \text { mechanical pain threshold (mechanische Schmerz- } \\
\text { schwelle), VDT vibration detection threshold (Vibrationsempfinden), } M C I \text { mild cognitive impairment } \\
\text { (leichtes kognitives Defizit) }\end{array}$} \\
\hline
\end{tabular}

signifikanten Dosisunterschiede zwischen den Gruppen. In der Gruppe mit normaler Kognition erhielten die Patienten im Mittel \pm SD 1,3 $\pm 0,5$ Schmerzmedikamente, in der Gruppe mit milder kognitiver Beeinträchtigung 2,0 0 und in der Gruppe mit Verdacht auf Demenz 2,0 $\pm 0,9$ Schmerzmedikamente. Als Nichtopioid-Analgetika wurden Ibuprofen (1200 bis $1800 \mathrm{mg} / \mathrm{Tag}$ ) und Metamizol (1500 bis $4000 \mathrm{mg} / \mathrm{Tag}$ ) und in je einem Fall Flupirtin ( $200 \mathrm{mg} / \mathrm{Tag})$ oder Diclofenac (150 mg/Tag) verordnet. $47 \%$ der Patienten wurden zusätzlich mit Opioiden versorgt (normale Kognition: $17 \%$ mit Opioid; milde kognitive Beeinträchtigung: 75\%; Verdacht auf De- menz: 56\%). Ein Patient mit chronischen Schmerzen erhielt seine Vormedikation Hydromorphon $24 \mathrm{mg} / \mathrm{Tag}$. Die anderen Patienten erhielten Tilidin (100 bis $300 \mathrm{mg} / \mathrm{Tag}$ ).

\section{Schmerzangabe mittels numerischer Ratingskala (NRS)}

Die mittlere postoperative Schmerzintensität (NRS; MW \pm SD) aller Patienten betrug $4,0 \pm 1,6$. Die Patienten mit normaler Kognition zeigten eine mittlere postoperative Schmerzstärke von 4,8 $\pm 1,8$ (NRS), die Patienten mit leichter kognitiver Beeinträchtigung von $3,3 \pm 1,5$ und Patienten mit Verdacht auf Demenz von 3,8 $\pm 1,3$. Am ersten postoperativen Tag lag der NRS-Mittelwert \pm SD aller Patienten bei $4,1 \pm 1,8$, am zweiten postoperativen Tag bei 3,5 $\pm 0,7$ und am dritten postoperativen Tag bei $4,0 \pm 1,4$. Die berichteten Schmerzintensitäten unterschieden sich nicht zwischen den Kognitionsgruppen und den ersten drei postoperativen Behandlungstagen (ANOVA; $p=$ n.s.).

\section{Schmerzempfindungsskala (SES)}

Der Summenscore für die affektiven Deskriptoren betrug im Mittel \pm SD 15,3 \pm 10,9, für die sensorischen Deskriptoren 10,7 $\pm 4,9$. Es konnten keine statistisch signifikanten Unterschiede für die sensorischen oder affektiven Summenscores der Schmerzempfindungsskala nachgewiesen werden (ANOVA; $p=$ n. s.).

Für die Faktorenanalyse der SES-Summenscores für den sensorischen Teil der Schmerzempfindungsskala ergab sich mit einem dreifaktoriellen Modell die beste Varianzaufklärung (74,4\%). Hier brachte der Faktor "oberflächlicher Schmerz" mit den dazugehörigen Begriffen "schneidend", "stechend", "heiß" und "durchstoßend" 


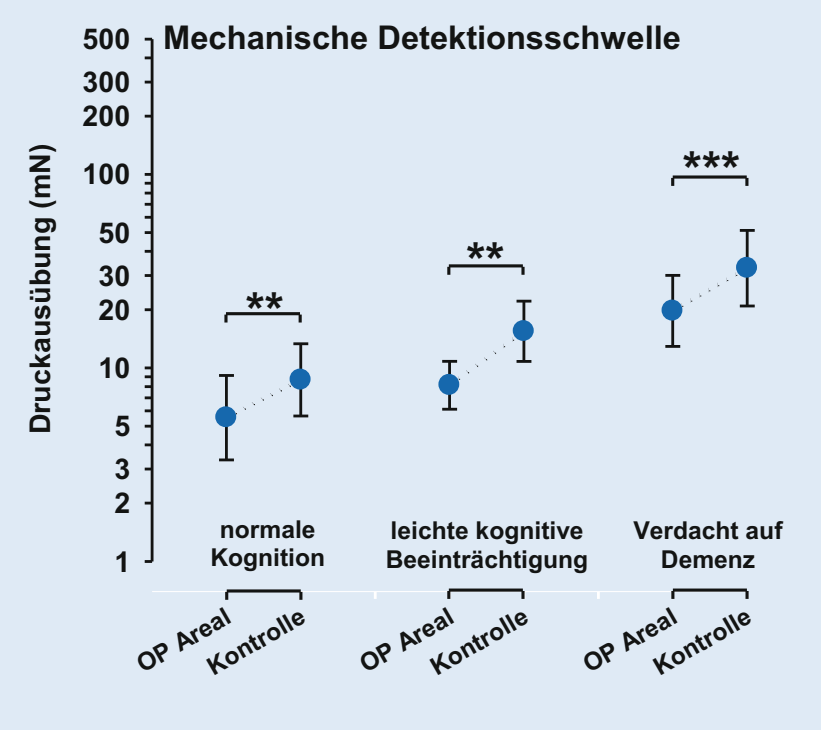

Abb. $1 \Delta$ Wahrnehmungsschwelle für leichte Berührungsreize mit "Von Frey"-Filamenten. Die Abbildung zeigt eine signifikante Abnahme (Sternchen: hoch signifikant) der Wahrnehmungsschwelle im Operationsgebiet - verglichen mit der nicht operierten, spiegelbildlichen Gegenseite. Die Unterschiede zwischen den Kognitionsgruppen waren nicht signifikant

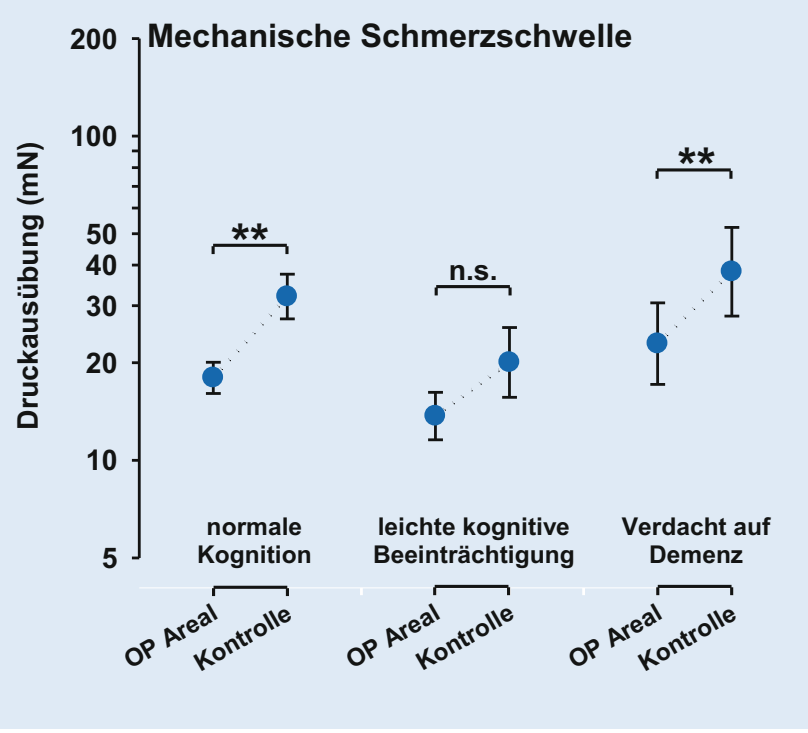

Abb. $2 \Delta$ Schmerzschwelle für punktförmige Nadelreize. Es zeigen sich signifikant (Sternchen: hoch signifikant) niedrigere Schmerzschwellen für Nadelreize über dem operierten Gebiet. Die Unterschiede zwischen den Kognitionsgruppen waren nicht signifikant. n.s. nicht signifikant
29,7\% Varianzaufklärung, der Faktor "Tiefenschmerz" mit den Begriffen "klopfend", "brennend" und "pochend" ergab 24,6\% sowie der Faktor "intensiver Schmerz oberflächlich und tief" mit den Begriffen "reißend" und "glühend" insgesamt 20,1\% Varianzaufklärung. Bezüglich der Aufklärung an der Gesamtvarianz der Faktorenanalyse des affektiven Teiles der Schmerzempfindungsskala wurde mittels eines Zweifaktoren-Modells eine Prozentzahl von 81,5 erreicht. Faktor 1 lässt sich hier als „allgemeine affektive Attribute" ("quälend", "grausam", "unerträglich“, ,"mörderisch“, ,elend“, ,"schauderhaft", "scheußlich", "marternd", ,furchtbar", ," heftig") beschreiben und Faktor 2 als „affektives Attribut mit motorisch hemmender Assoziation“ (,lähmend"). Für den Faktor 1 ergab sich eine Varianzaufklärung von 59,1\%, für den Faktor 2 von 22,4\%.

\section{Kognitive Fähigkeiten der Patienten}

Der mittlere DemTect-Score $( \pm S D)$ betrug $10,1 \pm 4,5$ Punkte. Von 19 Patienten lagen bei sechs $(31,6 \%)$ normale kognitive Fähigkeiten vor, bei vier Patienten (21,1\%) ergaben sich Hinweise auf leichte kognitive Einschränkungen, bei neun Patienten $(47,4 \%)$ ergab sich der Verdacht auf
Vorliegen einer Demenz. Das Fehlen von Interaktionen in der ANOVA weist darauf hin, dass die Zugehörigkeit zu einer Kognitionsgruppe unabhängig vom Alter der Patienten war.

\section{Postoperatives Schmerzempfinden und QST}

Die postoperative Schmerzempfindlichkeit - gemessen mittels QST - unterschied sich nicht zwischen den Kognitionsgruppen (• Tab. 2, Abb. 1, 2, 3 und 4). Die QSTParameter Druckschmerzschwelle (Tiefenschmerzempfindlichkeit; PPT), oberflächlicher mechanischer Schmerz nach Nadelreiz (MPT) und die oberflächliche Sensibilität für leichte Berührungsreize (MDT) zeigten jedoch hoch signifikante Unterschiede zwischen der operierten Seite und der gesunden Seite (ANOVA PPT: $F=47,9, p<0,001 ; \mathrm{MPT}: \mathrm{F}=20,5$, $p<0,001$; MDT: $\mathrm{F}=49,6, p<0,001)$. Für alle Parameter zeigte sich über der operierten Seite eine signifikant gesteigerte Empfindlichkeit. Für das Vibrationsempfinden (VDT) konnten keine Unterschiede zwischen operierter und gesunder Extremität nachgewiesen werden (ANOVA; $p=$ n.s.). Die Ergebnisse sind in - Tab. 2, sowie den $\bullet$ Abb. 1, 2, 3 und 4 dargestellt.

\section{Diskussion}

Wesentliche Befunde der vorliegenden Studie weisen darauf hin, dass sich das postoperative Schmerzerleben zwischen Patienten mit normaler und deutlich eingeschränkter Kognition nicht unterscheidet. Speziell die Wahrnehmung evozierter Schmerzreize im Rahmen der quantitativen sensorischen Testung zeigte keine Unterschiede zwischen den Kognitionsgruppen. Unmittelbar nach Operation waren die affektiven Schmerzqualitäten stärker als die sensorischen ausgeprägt und nahmen im Verlauf des zweiten und dritten postoperativen Tags deutlich ab. Die quantitative sensorische Testung zeigte mechanische Hyperalgesien im operierten Areal, die mit dem Konzept einer zugrundeliegenden peripheren und am ehesten sekundären zentralen Sensibilisierung der Gewebe nach Operation vereinbar sind.

\section{Postoperative Durchführbarkeit kognitiver und sensorischer Tests nach Femurfraktur}

Wie wichtig es ist, das Ausmaß postoperativer Schmerzen systematisch zu erfassen, wurde zuletzt in einer großen Kohor- 


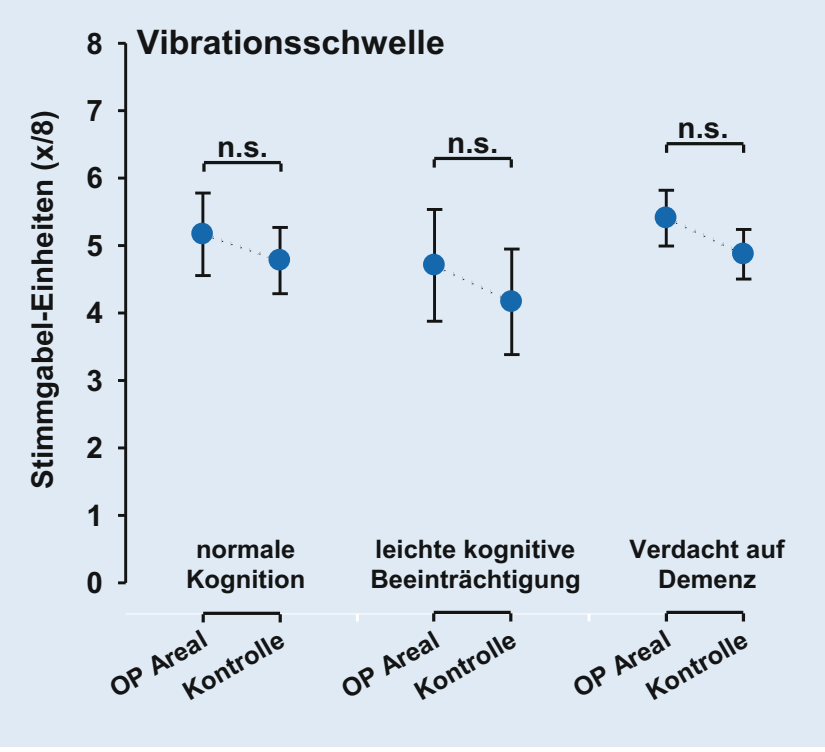

Abb. 3 ॥ Wahrnehmungsschwelle für Vibrationsreize mit einer $64 \mathrm{~Hz}$ Stimmgabel. Es finden sich keine Unterschiede zwischen den Testorten oder Kognitionsgruppen. n.s. nicht signifikant

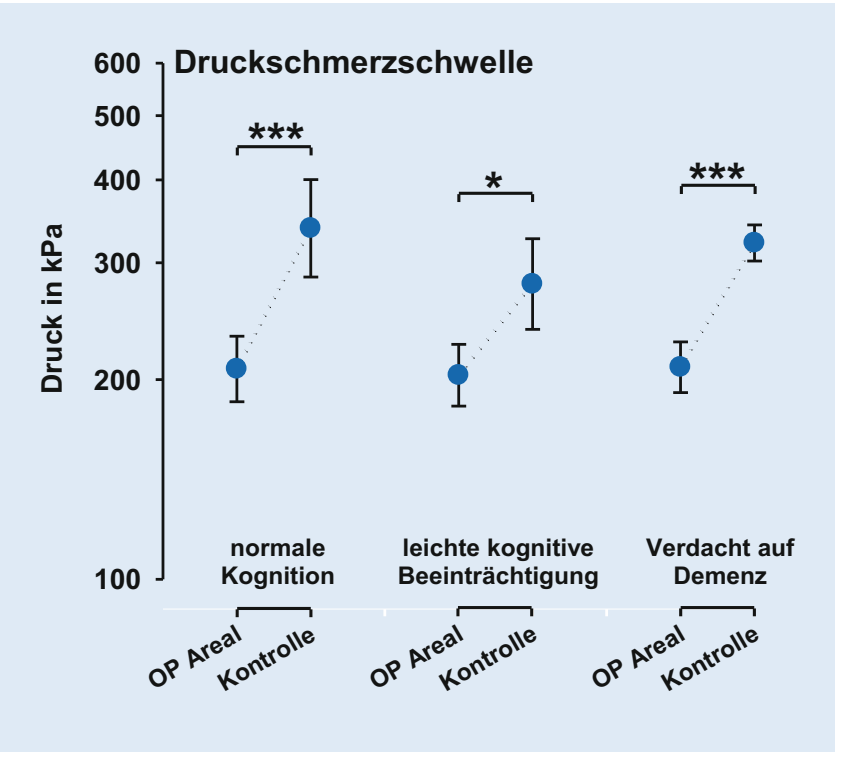

Abb. $4 \Delta$ Druckschmerzschwelle. Es zeigen sich signifikant niedrigere (Sternchen) Druckschmerzschwellen über dem operierten Gebiet. Die Unterschiede zwischen den Kognitionsgruppen waren nicht signifikant tenstudie deutlich, in der die Intensität der Schmerzen mittels numerischer Ratingskala am ersten postoperativen Tag bei 179 verschiedenen Op.-Prozeduren erfasst wurde. Interessant war, dass gerade bei "kleineren“ Eingriffen wie zum Beispiel Appendektomie, Tonsillektomie und Hämorrhoidektomie ein unerwartet hoher postoperativer Schmerzlevel vorlag [16].

In der vorliegenden Studie war es möglich, unabhängig von Attributen wie Alter oder kognitiven Fähigkeiten, einfachere Befragungen wie die Erfassung einer numerischen Schmerzschätzung durchzuführen. Der DemTect als bereits im klinischen Alltag etabliertes Instrument zur Einschätzung der kognitiven Fähigkeiten eines Patienten konnte hier ohne Einschränkungen bei allen Patienten angewendet werden. Auch bei den differenzierteren Fragebögen wie der Schmerzempfindungsskala waren die Patienten mit leicht eingeschränkten kognitiven Fähigkeiten und mit Verdacht auf Demenz in der Lage, ihr sensorisches und affektives Schmerzerleben genau zu differenzieren. Diese Fähigkeit zur Schmerzbeurteilung spiegelte sich auch bei der Untersuchung mittels QST wider. Auch hier kam es weder zu alters- noch kognitionsbedingten Schwierigkeiten bei der Durchführung. Allen Patienten war es postoperativ möglich, die vorgesehenen Untersuchungen durchzuführen. Dies bestätigt frühere Untersuchungen, die nachweisen, dass von bis zu mittelgradig dementen Patienten Berührungsreize und Schmerzreize differenziert beurteilt werden können [3, 14]. Diese Befunde belegen die Indikation für eine vorausschauend geplante und regelhafte postoperative Schmerzerhebung (z.B. NRS) zur Sicherstellung einer angemessenen pflegerischen Betreuung und Schmerzbehandlung [24, 35].

\section{Postoperatives Schmerzerleben und medikamentöses Management}

In der hier vorliegenden Studie zeigte sich kein signifikanter Zusammenhang zwischen postoperativ berichteter Schmerzstärke und kognitivem Status bei vergleichbarer analgetischer Therapie. Das postoperative Schmerzempfinden von $\mathrm{Pa}$ tienten mit kognitiver Beeinträchtigung unterschiedlichen Grades und kognitiv unbeeinträchtigten Patienten unterschied sich nicht signifikant. Auffällig war, dass anders als in der Literatur berichtet die Gruppen mit kognitivem Defizit häufiger Opioide in Ergänzung zu Nichtopioiden erhielten. Die kognitiven Einschränkungen waren also in unserem Kollektiv kein Prädiktor eines eingeschränkten Schmerzmanagements, was wir auf die etablierten
Schmerzstandards im Studienzentrum zurückführen.

\section{Postoperatives affektives und sensorisches Schmerzerleben}

Der Kognitionsstatus hatte keinen Einfluss auf das beschriebene affektive oder sensorische Schmerzerleben. Vor diesem Hintergrund erscheint die aus der Literatur bekannte Versorgungsrealität problematisch, die zeigt, dass demente Patienten weniger Schmerzmittel erhalten als solche mit normalen kognitiven Fähigkeiten [6, 30], auch im postoperativen Setting [42]. Eine Ursache könnte eine unzureichende und inadäquate Schmerzeinschätzung bei Patienten mit Demenz durch die betreuenden Ärzte und/oder das Pflegepersonal sein [33]. Zum anderen könnte eine unzureichende oder auch missverständliche Artikulation der Schmerzen durch die Patienten selbst eine Rolle spielen [24]. Schmerzäußerungen beispielsweise durch Vokalisationen werden häufig als postoperatives Delir oder Agitation fehlinterpretiert und könnten statt zu einer adäquaten Schmerztherapie zu sedierenden Maßnahmen wie der Gabe von Neuroleptika führen [19]. 
"Sensory profiling“ mittels QST nach operativer Versorgung einer Femurfraktur

In der quantitativen sensorischen Testung (QST) zeigte sich, dass die operierte Seite im Vergleich zur Gegenseite eine Überempfindlichkeit für mechanische Reize aufwies. Nur über dem operierten Gebiet zeigten sich eine Druckhyperalgesie sowie eine Nadelreiz-Hyperalgesie. Dieser Befund unterschied sich von Ergebnissen der Gruppe von Kehlet, die eine erhöhte Tiefensensibilität, nicht aber eine Hyperalgesie für kutane Reize finden konnten [1]. Im Unterschied zu unserer Studie wurde für die kutane Reizung elektrischer Strom verwendet, der unter Durchdringung der Haut ebenfalls tiefere Gewebeschichten erreichte. Interessanterweise zeigte unsere Studie auch für nichtschmerzhafte Reize mit "von Frey"-Filamenten eine Hyperästhesie, die über A-beta-Fasern vermittelt wird, während das ebenfalls peripher über A-beta-Fasern vermittelte Vibrationsempfinden sich nicht zwischen der Op.-Seite und dem unbehandelten Kontrollareal unterschied. Dieser Befund weist darauf hin, dass leichte taktile Reize und Vibrationsreize trotz gleichem peripherem Fasersystem möglicherweise zentral über zwei verschiedene Projektionswege zum Thalamus weitergeleitet werden. Möglicherweise wird nur ein System im Rahmen des postoperativen Geschehens sensibilisiert, während das andere unbeeinflusst bleibt. Eine weitere Erklärung könnte sein, dass das Vibrationsempfinden mehr die Sensibilität tieferer Gewebe (protopathisch) reflektiert, während die oberflächliche Berührungsempfindlichkeit der Haut (epikritisch) besser durch den Parameter MDT repräsentiert wird. MDT mag damit stärker durch Umwelteinflüsse und Alterung der Haut als VDT beeinflusst werden. Zusätzlich mag es für die mechanische Detektionsschwelle mit von FreyFilamenten eine Rolle spielen, dass die Erfassung dieses Parameters ein diffiziler Akt ist, der mehr als die Bestimmung der Vibrationsschwelle vom Kognitionsstatus abhängen könnte, was sich in einem diskreten Trend zu höheren Schwellen bei abnehmendem Kognitionsstatus andeutete. Auch sollte beachtet werden, dass VDT gegenüber dem DFNS-QST-Protokoll nicht über einer knöchernen Prominenz erfasst wurde.

\section{Vom klinischen Zeichen zum neurobiologischen Mechanismus}

In der Literatur wurde bereits in Tierexperimenten gezeigt, dass nach bestimmten konditionierenden Reizen unterschiedliche neurobiologische Schmerzmechanismen induziert werden können. Im Rahmen von Entzündungsmodellen nach Injektionen von beispielsweise Capsaicin konnte eine gesteigerte Aktivität von zentralen "wide dynamic range"-Neuronen (WDR-Neuronen) im Hinterhorn des Rückenmarks nachgewiesen werden. Kardinalsymptom dieser zentralen Sensibilisierung ist eine mechanische Hyperalgesie für Nadelreize (Pinprick-Hyperalgesie) [43]. Hier können aber auch andere zentrale Mechanismen eine Rolle spielen [32].

Die Hyperalgesie für Nadelreize über der nicht durch die Operation betroffenen Haut muss als auf die Haut übertragene Hyperalgesie interpretiert werden (,referred pain“-Konzept) [18]. Dieses Phänomen muss per Definition als zentral vermittelt angesehen werden - am ehesten im Sinn einer sekundären Hyperalgesie nach Sensibilisierung von WDR-Neuronen im Hinterhorn des Rückenmarks. Interessanterweise findet sich im Op.-Bereich auch eine taktile Hyperästhesie für leichte Berührungsreize („von Frey“-Filamente), die am ehesten ebenfalls zentral vermittelt ist. Dieses Phänomen einer sekundären mechanischen Hyperästhesie wurde bisher nicht beschrieben. In der Literatur wird in experimentellen Schmerzmodellen nach Capsaicin-Injektion das Phänomen einer sekundären taktilen Hypoästhesie bei gleichzeitiger Nadelreiz-Hyperalgesie beschrieben [28]. In diesem Modell liegt jedoch kein so ausgeprägter peripherer nozizeptiver Input vor wie nach der hier beschriebenen operativen Versorgung einer Femurfraktur. Neben den zentralen neuroplastischen Veränderungen im Schmerzsystem können auch periphere Sensibilisierungsprozesse für die lokal gesteigerte Schmerzempfindlichkeit verantwortlich sein. In dem oben beschriebenen Entzündungsmodell nach intrakutaner CapsaicinGabe kommt es primär zu einer periphe- ren Sensibilisierung von Nozizeptoren am Ort der Injektion, die zu einer lokalen Hitzehyperalgesie führen kann. Der TRPV1lonenkanal (Vanilloid-Rezeptor 1) hat eine wesentliche Bedeutung für die Entstehung der Hitzehyperalgesie, da er über eine spezifische Bindungsstelle (z. B. für Capsaicin) verfügt, zum anderen aber auch über eine erhöhte Temperatur aktiviert wird. Auch nach Schnittverletzungen kommt es zu einer lokalen Überempfindlichkeit gegenüber Hitzereizen (primäre Hitzehyperalgesie). Dies wurde nicht nur im Tiermodell bei Mäusen nachgewiesen [34], sondern auch im humanen Surrogatmodell [9]. Verantwortlich hierfür ist eine gesteigerte $\mathrm{Er}$ regbarkeit von $\mathrm{A} \delta$ - und C-Faser-Nozizeptoren. Möglicherweise kann die Absenkung der Erregungsschwelle nozizeptiver C-Fasern zu einer erleichterten Aktivierbarkeit führen und damit den Ruheschmerz triggern. Als weiteres Symptom einer peripheren Sensibilisierung wird in der Literatur eine Druckhyperalgesie beschrieben $[21,26]$. Im getesteten Patientenkollektiv konnte dieser Befund einer gesteigerten Druckempfindlichkeit tieferer Gewebe reproduziert werden, was indirekt auf das wahrscheinliche Vorliegen einer primären, postoperativen peripheren Sensibilisierung hinweist. Diese und der damit verbundene gesteigerte sensorische Input führen vermutlich sekundär zu einer zentralen Sensibilisierung oder anderen zentral neuroplastischen Veränderungen, die ursächlich für die oben beschriebene Nadelreizhyperalgesie sein könnten.

\section{Limitierungen}

Aus der vorliegenden Studie lassen sich keine Generalisierungen vornehmen, da im Rahmen dieser Pilotstudie nur eine kleine Patientenpopulation untersucht wurde $(n=19)$. Es kann letztlich nicht mit Sicherheit ausgeschlossen werden, dass die fehlenden Gruppenunterschiede durch diese kleine Fallzahl (zu geringe statistische Power) bedingt sind. Wünschenswert wäre eine Folgestudie mit größerer Fallzahl, um die hier gewonnenen Ergebnisse verifizieren zu können und mehr über das postoperative Schmerzerleben von Patienten mit eingeschränkten kognitiven Fähigkeiten zu erfahren. Dabei sollte auch beachtet werden, dass etwa die Dosie- 
rung von Schmerzmitteln wie Opioiden bestmöglich vergleichbar zwischen diesen Kognitionsgruppen ist. In unserer Studie wurden die Patienten mit normaler Kognition weniger häufig postoperativ mit Opioiden behandelt. Eine weitere Limitierung war die dem Eingriff geschuldete operationsnahe Erfassung der Vibrationsschwelle (VDT) über der Haut fernab von einer knöchernen Prominenz. Diese Abweichung vom Original-QST-Protokoll des DFNS führte zu VDT-Werten um 5/8, was sich jedoch nicht wesentlich von altersentsprechenden Referenzwerten etwa des Knöchels unterscheidet.

\section{Fazit und Ausblick}

Es zeigte sich, dass die postoperative Schmerzstärke innerhalb der Patientengruppe $(n=19)$ unabhängig vom kognitiven Status war. Im sensorischen und affektiven Schmerzerleben ergeben sich im SES-Fragebogen (Schmerzempfindungsskala) zwischen den untersuchten Patientengruppen mit normaler Kognition bis hin zum Verdacht auf Demenz keine signifikanten Unterschiede. Die quantitative sensorische Testung (QST) weist nur über dem operierten Areal eine mechanische Überempfindlichkeit aus. Konkret zeigte sich dort neben einer Nadelreizund Druckhyperalgesie eine taktile Hyperästhesie für "von Frey“-Filamente. Das Vibrationsempfinden unterschied sich nicht zwischen operierter Seite und dem Kontrollareal. Die gefundene Druckhyperalgesie im operierten Gebiet ist vereinbar mit dem Konzept einer peripheren Sensibilisierung im nozizeptiven System. Diese periphere Sensibilisierung induziert möglicherweise sekundär auch eine zentrale Sensibilisierung. Passend zu dieser Annahme findet sich das klinische Zeichen einer Nadelreizhyperalgesie über der Haut des operierten Gebiets, die am ehesten zentral vermittelt ist - ebenso wie eine taktile Hyperästhesie, die nur zentralnervös erklärt werden kann und in dieser Form noch nicht beschrieben ist.

Insgesamt weisen alle Befunde dieser Feasibility-Studie darauf hin, dass Betroffene mit kognitiven Defiziten ein unverändertes Schmerzerleben und auch eine vergleichbare Reagibilität ihres nozizeptiven Systems aufweisen. Hieraus leitet sich der auch medizinethisch bedeutsame Anspruch ab, die gleichen hohen Ansprüche an eine optimale postoperative Schmerztherapie auch für Menschen mit Demenz zu stellen. Prinzipiell sind auch Studien mit anderen operativen Prozeduren für Menschen mit kognitivem Defizit wünschenswert.

\section{Korrespondenzadresse}

\section{Dr. med. Henning Cuhls}

Klinik und Poliklinik für Palliativmedizin, Universitätsklinikum Bonn

Bonn, Deutschland

henning.cuhls@uni-bonn.de

Danksagung. Unser Dank gilt den Patienten, die sich für die Erfassung der hier vorgestellten Daten zur Verfügung gestellt haben. R. Rolke wird gefördert mit Mitteln des Interdisziplinären Zentrums für Klinische Forschung in der Medizinischen Fakultät der RWTH Aachen (IZKF TN1-6/IA 532006) und durch das BMBF-geförderte Bio2Treat-Konsortium (BMBFFörderkennzeichen 13GW0334B).

Funding. Open Access funding enabled and organized by Projekt DEAL.

\section{Einhaltung ethischer Richtlinien}

Interessenkonflikt. J.F. Wagner, H. Cuhls, M. Mücke, R. Conrad, L. Radbruch und R. Rolke geben an, dass kein Interessenkonflikt besteht.

Für diesen Beitrag wurden von den Autoren keine Studien an Menschen oder Tieren durchgeführt. Für die aufgeführten Studien gelten die jeweils dort angegebenen ethischen Richtlinien.

Open Access. Dieser Artikel wird unter der Creative Commons Namensnennung 4.0 International Lizenz veröffentlicht, welche die Nutzung, Vervielfältigung, Bearbeitung, Verbreitung und Wiedergabe in jeglichem Medium und Format erlaubt, sofern Sie den/die ursprünglichen Autor(en) und die Quelle ordnungsgemäßnennen, einen Link zur Creative Commons Lizenz beifügen und angeben, ob Änderungen vorgenommen wurden.

Die in diesem Artikel enthaltenen Bilder und sonstiges Drittmaterial unterliegen ebenfalls der genannten Creative Commons Lizenz, sofern sich aus der Abbildungslegende nichts anderes ergibt. Sofern das betreffende Material nicht unter der genannten Creative Commons Lizenz steht und die betreffende Handlung nicht nach gesetzlichen Vorschriften erlaubt ist, ist für die oben aufgeführten Weiterverwendungen des Materials die Einwilligung des jeweiligen Rechteinhabers einzuholen.

Weitere Details zur Lizenz entnehmen Sie bitte der Lizenzinformation auf http://creativecommons.org/ licenses/by/4.0/deed.de.

\section{Literatur}

1. Aasvang EK, Werner MU, Kehlet H (2014) Assessment of deep tissue hyperalgesia in the groin-a method comparison of electrical vs. pressure stimulation. Acta Anaesthesiol Scand 58:986-996

2. Anonymous (2000) A fifth amendment for the declaration of Helsinki. Lancet 356:1123

3. Benedetti F, Arduino C, Vighetti S et al (2004) Pain reactivity in Alzheimer patients with different degrees of cognitive impairment and brain electrical activity deterioration. Pain 111:22-29

4. Chan AW, Macfarlane IA, Bowsher D et al (1992) Weighted needle pinprick sensory thresholds: a simple test of sensory function in diabetic peripheral neuropathy. J Neurol Neurosurg Psychiatry 55:56-59

5. Fagius J, Wahren LK (1981) Variability of sensory threshold determination in clinical use. J Neurol Sci 51:11-27

6. Feldt KS, Ryden MB, Miles S (1998) Treatment of pain in cognitively impaired compared with cognitively intact older patients with hip-fracture. JAm Geriatr Soc 46:1079-1085

7. Ferrell BA, Ferrell BR, Rivera L (1995) Pain in cognitively impaired nursing home patients. J Pain Symptom Manage 10:591-598

8. Field L (1996) Are nurses still underestimating patients' pain postoperatively? Br J Nurs 5:778-784

9. Fimer I, Klein T, Magerl W et al (2011) Modalityspecific somatosensory changes in a human surrogate model of postoperative pain. Anesthesiology 115:387-397

10. Fisher SE, Burgio LD, Thorn BE et al (2002) Pain assessment and management in cognitively impaired nursing home residents: association of certified nursing assistant pain report, minimum data set pain report, and analgesic medication use. JAm Geriatr Soc 50:152-156

11. Friedman SM, Menzies IB, Bukata SV et al (2010) Dementia and hip fractures: development of a pathogenic framework for understanding and studying risk. Geriatr Orthop Surg Rehabil 1:52-62

12. Frietsch T, Schuler M, Adler G (2014) Der demenzkranke Patient - Was ist beim anästhesiologischen Management zu beachten? Anasthesiol Intensivmed Notfallmed Schmerzther 49:220-229

13. Fruhstorfer $\mathrm{H}$, Gross W, Selbmann $\mathrm{O}$ (2001) von Frey hairs: new materials for a new design. Eur J Pain 5:341-342

14. Gagliese L, Gauthier LR, Narain N et al (2018) Pain, aging and dementia: towards a biopsychosocial model. Prog Neuropsychopharmacol Biol Psychiatry 87:207-215

15. Geissner E (1995) The pain perception scale-a differentiated and change-sensitive scale for assessing chronic and acute pain. Rehabilitation 34:XXXV-XLIII

16. Gerbershagen HJ, Aduckathil S, Wijck AJM et al (2013) Pain intensity on the first day after surgery: a prospective cohort study comparing 179 surgical procedures. Anesthesiology 118:934-944

17. Goldberg JM, Lindblom U (1979) Standardised method of determining vibratory perception thresholds for diagnosis and screening in neurological investigation. J Neurol Neurosurg Psychiatry 42:793-803

18. Graven-Nielsen T (2006) Fundamentals of muscle pain, referred pain, and deep tissue hyperalgesia. Scand J Rheumatol Suppl 122:1-43

19. Helvik AS, Bergh S, Saltyte Benth J et al (2021) Pain in nursing home residents with dementia and its 
association to quality of life. Aging Ment Health. https://doi.org/10.1080/13607863.2021.1947968

20. Kalbe E, Kessler J, Calabrese P et al (2004) DemTect: a new, sensitive cognitive screening test to support the diagnosis of mild cognitive impairment and early dementia. Int J Geriat Psychiatry 19:136-143

21. Kilo S, Schmelz M, Koltzenburg M et al (1994) Different patterns of hyperalgesia induced by experimental inflammation in human skin. Brain 117(2):385-396

22. Klopfenstein CE, Herrmann FR, Mamie Cetal (2000) Pain intensity and pain relief after surgery. A comparison between patients' reported assessments and nurses' and physicians' observations. Acta Anaesthesiol Scand 44:58-62

23. Kosek E, Ekholm J, Hansson P (1999) Pressure pain thresholds in different tissues in one body region. The influence of skin sensitivity im Druckure algometry. Scand J Rehabil Med 31:89-93

24. Krupic F, Sadic S, Seffo N et al (2018) Experience of registered nurses in assessing postoperative pain in hip fracture patients with dementia. Med Glas (Zenica) 15:75-80

25. Kunz R (2012) Deutlich erhöhte Prävalenz chronischer Schmerzen. ProCare 17:24-30

26. Latremoliere A, Woolf CJ (2009) Central sensitization: a generator of pain hypersensitivity by central neural plasticity. J Pain 10:895-926

27. Lynch EP, Lazor MA, Gellis JE et al (1998) The impact of postoperative pain on the development of postoperative delirium. Anesth Analg 86:781-785

28. Magerl W, Treede R-D (2004) Secondary tactile hypoesthesia: a novel type of pain-induced somatosensory plasticity in human subjects. Neurosci Lett 361:136-139

29. Morrison RS, Magaziner J, Mclaughlin MA et al (2003) The impact of post-operative pain on outcomesfollowing hip fracture. Pain 103:303-311

30. Morrison RS, Siu AL (2000) A comparison of pain and its treatment in advanced dementia and cognitively intact patients with hip fracture. J Pain Symptom Manage 19:240-248

31. Mücke M, Cuhls H, Radbruch L et al (2021) Quantitative sensory testing (QST). English version. Schmerz 35(3):153-160. https://doi.org/ 10.1007/s00482-015-0093-2

32. Mücke M, Cuhls H, Radbruch Let al (2014) Evidence of heterosynaptic LTD in the human nociceptive system: superficial skin neuromodulation using a matrix electrode reduces deep pain sensitivity. PLoSOne 9:e107718

33. Pesonen A, Kauppila T, Tarkkila P et al (2009) Evaluation of easily applicable pain measurement tools for the assessment of pain in demented patients. Acta Anaesthesiol Scand 53:657-664

34. Pogatzki-Zahn EM, Shimizu I, Caterina $\mathrm{M}$ et al (2005) Heat hyperalgesia after incision requires TRPV1 and is distinct from pure inflammatory pain. Pain 115:296-307

35. Rasmussen LS, Jorgensen CC, Kehlet H (2016) Enhanced recovery programmes for the elderly. Eur J Anaesthesiol 33:391-392

36. Rodgers A, WalkerN, Schug Setal (2000) Reduction of postoperative mortality and morbidity with epidural or spinal anaesthesia: results from overview of randomised trials. BMJ321:1493

37. Rolke R, Andrews Campbell K, MagerlWetal (2005) Deep pain thresholds in the distal limbs of healthy human subjects. Eur J Pain 9:39-48

38. Rolke R, Baron R, Maier C et al (2006) Quantitative sensory testing in the German research network on neuropathic pain (DFNS): standardized protocol and reference values. Pain 123:231-243

\section{Postoperative pain experience after proximal femur fracture in dementia}

Background: The present study aimed to assess the postoperative pain experience in cognitive deficit patients with special reference to sensory or affective pain quality. Methods: Nineteen patients with normal cognition up to cognitive impairments according to the DemTect screening-tool were studied regarding their postoperative pain experience after proximal femur fracture. The numerical rating scale (NRS), the cognitive DemTect questionnaire, the pain sensation questionnaire (SES), and a quantitative sensory test (QST) were used as examination instruments.

Results: The mean \pm SD age of the patients was $83.8 \pm 10.0$ years. Of the 19 patients, $6(31.6 \%)$ had normal cognitive abilities. In 4 patients (21.1\%) there were indications of mild cognitive impairments, and in 9 patients (47.4\%) the suspicions of the presence of dementia arose. The mean postoperative pain intensity (NRS) was 4.0 (1.6). With comparable analgesic therapy, the reported pain intensities did not differ between the three patient groups with different cognitive impairments and the first three postoperative treatment days. There were no statistically significant differences between the groups for the sensory or affective total scores of the pain sensation scale. The QST parameters deep pain (PPT), superficial mechanical pain after needle stimulation (MPT), and the superficial sensitivity to light touch stimuli (MDT) showed a significantly increased sensitivity of the operated side. For the sensation of vibration (VDT) no differences between operated and healthy extremities could be proven. Discussion: The postoperative pain experience does not differ between patients with normal and limited cognition. The quantitative sensory testing showed mechanical hyperalgesia in the operated area. The study points to the importance of adequate postoperative pain management even in those with dementia.

\section{Keywords}

Pain sensation scale · Quantitative sensory testing · Somatosensory nervous system · Sensory examination. Geriatrics

39. Rolke R, Magerl W, Campbell KA et al (2006) Quantitative sensory testing: a comprehensive protocol for clinical trials. Eur J Pain 10:77-88

40. Rond ME, Wit R, Dam FS et al (2000) A pain monitoring program for nurses: effects on communication, assessment and documentation of patients' pain. J Pain Symptom Manage 20:424-439

41. Rösler A, Krause T, Niehuus Cet al (2009) Dementia as a cofactor for geriatric rehabilitation-outcome in patients with osteosynthesis of the proximal femur: a retrospective, matched-pair analysis of 250 patients. Arch Gerontol Geriatr 49:e36-39

42. Sieber FE, Mears S, Lee Het al (2011) Postoperative opioid consumption and its relationship to cognitive function in older adults with hip fracture. J Am Geriatr Soc 59:2256-2262

43. Simone DA, Sorkin LS, Oh U et al (1991) Neurogenic hyperalgesia:central neural correlates in responses of spinothalamic tract neurons. J Neurophysiol $66: 228-246$ 